\title{
Analysis on Perioperative Nursing of Urological Laparoscopic Surgery
}

\author{
Lingbo Peng* \\ Longnan Hospital of Daqing Oil Field General Hospital Group (Fifth Affiliated Hospital of Qiqihar Medical University), \\ Daqing, Heilongjiang 163001, China
}

ABSTRACT Objective: To summarize the perioperative nursing experience of urological laparoscopic surgery. Method: Make retrospective summary and analysis of the 192 laparoscopic surgery cases treated in our department from March 2011 to May 2015. Result: Due to the close cooperation of the medical staff, the 192 patients recovered well and were all cured and discharged from hospital. Conclusion: Meticulous, careful and comprehensive perioperative nursing plays an important positive role in the early recovery of the patients.

\author{
KEYWORDS \\ Laparoscopic \\ Urological surgery \\ Perioperative nursing
}

\section{Introduction}

Since the first successful laparoscopic nephrectomy surgery by Clayman of America in 1990, laparoscopy has attracted wide attention of urology surgery and become the pioneer of modern urology laparoscopic surgery [1]. After more than 10 years of development, the application of laparoscopy in urology surgery has been gradually made known to the medical staff of all levels in hospitals and among the patients in China. Compared with traditional surgery, it has such advantages as small wound surface, slight injury, less bleeding and quick recovery, so it has been favored even more by both doctors and patients. However, with the gradual popularization of laparoscopic techniques, all kinds of complications have also increased [2]. In order to avoid or reduce the complications of laparoscopic surgery, it is necessary to discover and deal with them immediately so as to relieve the suffering of the patients and help them recover as early as possible. Therefore, it is of great importance to strengthen perioperative nursing. The general nursing situation of the 192 cases of laparoscopic surgery

Copyright $\odot 2016$ Lingbo Peng

doi: $10.18686 /$ jn.v5i4.10

Received: August 23, 2016; Accepted: November 9, 2016; Published online: December 29, 2016

This is an open-access article distributed under the terms of the Creative Commons Attribution Unported License (http://creativecommons.org/ licenses/by-nc/4.0/), which permits unrestricted use, distribution, and reproduction in any medium, provided the original work is properly cited.

${ }^{*}$ Corresponding author: Longnan Hospital of Daqing Oil Field General Hospital Group (Fifth Affiliated Hospital of Qiqihar Medical University), Daqing, Heilongjiang 163001, China. E-mail: lingbo_dq@hotmail. com treated in our department from March 2011 to May 2015 is reported below.

\section{Data and methods \\ 2.1. General data}

This group includes 192 sample cases, including 104 males and 88 females with age ranging from 15 to 81 years old and the average age of 44.1 years old. Among them, 43 cases received adrenalectomy, 10 cases received nephrectomy, 15 cases received renal tumour enucleation, five cases received renal pedicle lymphatic disconnection, 27 cases received radical prostatectomy, 19 cases received radical nephroureterectomy, 22 cases received pyeloureterostomy, 25 cases received renal cyst unroofcuring, 19 cases received polycystic kidney unroofing and 7 cases received bilateral ligation of spermatic vein.

\subsection{Retroperitoneal approach}

Adopt general anesthesia on the uninjured side, cut a $1.5 \mathrm{~cm}$ small incision at $0.5 \mathrm{~cm}$ to $1 \mathrm{~m}$ under the costal margin of the posterior axillary line (Point A), and put a self-control balloon expander to expand the retroperitoneal for five minutes after the anesthetics penetrates into the retroperitoneal via blunt dissection. Puncture 2 $\mathrm{cm}$ above the spina iliac of the midaxillary line (B point) and under costal margin of the anterior axillary line (C point) to prepare passageway, penetrate $10 \mathrm{~mm}, 5 \mathrm{~mm}$ and $10 \mathrm{~mm}$ Trocar at points A, B and C separately, and then place operating apparatus and monitoring oscilloscope. Prepare pneumoperitoneum with $10-15 \mathrm{mmHg}$ $\mathrm{CO}_{2}$ and have operation under the surveillance of the TV screen. Approach it via abdominal cavity and adopt 
general anesthesia on horizontal position. The setting pressure of pneumoperitoneum pressure is 10 to 15 mmHg. Cut an annular cut of $1 \mathrm{~cm}$ below the umbilicus and inserts $10 \mathrm{~mm}$ Trocar and peritoneoscope, which is used to monitor the Trocar of other places and surgical instruments. Perform the surgery under the surveillance of the monitor.

\section{Nursing}

\subsection{Psychological nursing}

All patients expect to be well cared by the medical staff after being admitted to hospital and to get high quality treatment and care. It is also human nature for the patients to harbor some suspicion. How to do a good job out of psychological counselling for the patients? First of all, the nursing staff should gain the trust of patients step by step, establish a good doctor-patient relationship and help them have a simple understanding of the system and operating methods of the hospital and departments; face up to their illnesses correctly, stabilize their mood and comply with the medical care plan. Let the patients understand the characteristics of laparoscopic surgery and explain in detail about the operation procedures to the patients and their families. It is also a good way to invite a patient who has recovered to comfort the other patients, prepare everything well to make sure that the patients are comfortable and create a good environment to ensure the patients receive the operation with a good attitude, which is of great importance to the improvement of the success rate of the surgery [3].

\subsection{Pre-operative nursing}

In addition to the comprehensive routine inspection of the heart, liver, lung and kidney before the operation. Besides that, other inspection for existing diseases must be treated, for example, for diabetes, the blood glucose level should be controlled and for cardiovascular patients who take aspirin should discontinue the drug a week before the operation. Besides, patients with the smoking habit should quit smoking for a week. Penicillin or cephalothin skin test before the operation should be conducted, as required by the post-operation. Blood cross-match needed during the operation should also be done. Disinfect skin of the operative region and clean the intestine in morning or at night before the operation.

\subsection{Post-operative nursing}

\subsubsection{Close observation of the change of vital signs}

Conduct electrocardiograph monitoring, monitor the degree of blood oxygen saturation, measure the blood pressure, pulse and respiration every 15 to 30 minutes, and report abnormal changes in time to the doctors for treatment. Keep respiratory unobstructed, offer low-flow intermitted oxygen absorption and monitor change of the blood oxygen saturation at any time. Since retroperitoneal laparoscopic surgery is completed under $\mathrm{CO}_{2}$ insufflations which may cause hypercapnia and continues for a certain time after the operation, it is necessary to eliminate the accumulated $\mathrm{CO}_{2}$ through deep breath and acceleration of the autogenous regulation. Thus, it is essential to control the infusion speed and amount, and accurately record urine volume of 24 hours to prevent cardiopulmonary failure [4].

\subsubsection{Position}

Patients who are not fully awake should adopt recumbent position without pillow and tilt their heads to one side in order to keep respiratory tract unobstructed. The intraoral secretions should be closely observed and cleared to assist sputum excretion. It is advisable to encourage early ambulation of the patients, most of whom can get out of bed in two to three days.

\subsubsection{Post-operative monitoring}

It includes post-operative routine ECG monitoring, low flow oxygen inhalation, accurate record of urine volume of 24 hours, control of the total quantity of the infusion and dripping speed. Abnormal situation must be reported to the doctor on-time for timely treatment.

\subsubsection{Post-operative diet management}

After the patients regain conscious after laparoscopic surgery, they can restore diet if there is no maladjustment, by taking liquid food first, transiting to normal diet the next day and drinking more water. It is advised to avoid excitant food. Due to the carbon dioxide retention of laparoscopic surgery, it is appropriate to increase the intake of vegetables and fruits with high fiber; avoid eating food that will produce gas, such as sweet potato, beans, onions, etc. Intestinal surgery patients cannot take food before anus exhaust.

\subsubsection{Observation and nursing of complications Subcutaneous emphysema}

Retroperitoneal laparoscopic surgery adopts the method of filling the space of abdominal cavity. Strong gas dispersion capacity can directly permeate into the subcutaneous tissue or rise to the face from the outside of the pleura, which may easily form subcutaneous emphysema that can be totally absorbed into the blood and expelled out of the body through pulmonary respiration. However, it is necessary to give low-flow intermittent oxygen so as to raise the partial pressure of blood oxygen.

\section{Observation of the bleeding of puncture holes}

The bleeding of the puncture hole is caused by incomplete hemostasis or falloff of the titanium clamp. Pay attention to whether the hole puncture application oozes blood, and change it after being soaked through compression bandage [5]. 


\section{Observation of hypercapnia}

Since artificial pneumoperitoneum has certain influence on the circulation system and respiratory system, $\mathrm{CO}_{2}$ dispersion is widely absorbed into the blood (which causes the increase of $\mathrm{CO}_{2}$ in the blood to exceed the lung compensatory ability); it shows the symptoms of respiratory acidosis and leads to pulmonary embolism or cerebral embolism if it is really serious. Observe whether patients show the symptoms of fatigue, agitation and hypopnea after the operation. Give low-flow intermittent oxygen inhalation and keep respiratory tract unobstructed and encourage patients to effectively cough in order to improve the oxygen partial pressure and promote $\mathrm{CO}_{2}$ discharge. Continued high concentration of oxygen intake should be avoided, because excessive oxygen can inhibit the respiratory center and make breathing slow; this is not conductive to the discharge of $\mathrm{CO}_{2}$.

\section{Abdominal pain}

It is caused by the stimulation of the tension when the peritoneum that covers the surface of body organs and hides in the inside of the abdominal cavity is dragged and aerated. Besides, the $\mathrm{CO}_{2}$ filled in the abdominal cavity can function easily with water and form carbonic acid, which worsens the stimulation of the peritoneum and causes pain. In this situation, small doses of painkillers can be used. The symptoms of this group of seven cases of patients were relieved after using them.

\section{Retroperitoneum bleeding}

The reasons for bleeding include that renal artery sheath is damaged, adrenal venous is branched and the blood vessel titanium clamp falls off, etc.; in addition, due to the high pressure of pneumoperitoneum and retroperitoneal space, secondary hemorrhage can be caused. Closely observe abdominal symptoms, vital signs, and the quantity and quality of the drainage fluid; if the drainage fluid is bright red in color and the amount $>100 \mathrm{~mL} / \mathrm{h}$, report to the doctor in-time for timely treatment. One of the patients had secondary hemorrhage. Hence hemostatic drugs were used and the drawing of the retroperitoneal drainage tube was 10 days delayed.

\section{Treatment of acute gastric retention}

The common causes include that the bowel function is inhibited after anesthesia, which lead to the excessive pneumatosis in the enteric cavity or the stimulation of $\mathrm{CO}_{2}$ to diaphragm. Hence, at the end of the surgery, the abdomen should be squeezed to reduce the retention of $\mathrm{CO}_{2}$; getting out of bed as early as possible can promote intestinal peristalsis.

\subsection{Pain management}

The American Pain Society has proposed to make pain grade as the fifth vital sign following body tempera- ture, pulse, respiration and blood pressure [6]. The general post-operative pain is most violent two to 16 hours after the patient sobers up from the anesthesia and then it slowly mitigates and disappears after 72 hours. The pain can be alleviated by changing the position of the patient, using analgesia pump and taking painkillers.

\subsection{Health and discharge guidance}

Urge the patients to eat food with high heat, high protein and rich vitamins, encourage them to get out of bed as early as possible, explain to them self-care knowledge with drainage tube, the purpose of drug use and the matters needing attention as well as the related knowledge of the disease, so as to make the patients be familiar with self-care. Nephrectomy patients should take B ultrasound and renal function review three months after the operation; while the patients with renal cyst should take periodic review of $B$ ultrasound to confirm the efficacy of treatment.

\section{Discussion}

Retroperitoneal laparoscopic surgery has been widely used in urology, and the treatment is effective. Compared with traditional open surgery, it has the advantages of small trauma, rapid recovery, less bleeding and shorter hospitalization days, and is a safe and effective form of minimally invasive surgery. At present, laparoscopic surgery can be used in most urology surgeries. Due to its obvious advantages compared with traditional surgery, it is praised and preferred by the masses of medical staff in urology surgery. However, because of its more complex operation, it takes a long time for a urology surgeon to master it. So the proficiency of the performer and post-operative complications are negatively correlated. The close cooperation and careful nursing of the nursing staff can greatly reduce such factors. Key tasks during the nursing includes: (1) Do a good job with psychological nursing and make the patient and the family cooperate with the treatment. (2) Post-operative close observation of vital signs is the key; it can detect abnormal situations for timely treatment. The approach of retroperitoneal laparoscopic surgery is more direct, and the urological surgeons are familiar with the path, which brings little interference to the organs inside, does not infect the abdominal viscera, and reduces gastrointestinal reaction, postoperative abdominal infection and intestinal adhesion.

In short, although perioperative nursing of urology is complicated, as long as the nursing staff develop the habit of carefulness and rigorousness, have the attitude of evidence-based medicine, continuously strengthen business study and summarize the work experience, they can timely discover and deal with the problems. These reduce the occurrence and development of the complications, improve the success rate of the laparoscopic urology, reduce the 
pain of patients and help patients recover as early as possible.

\section{References}

1. Na Y. Laparoscopic surgery. Wu Jieping Urological Surgery. Shandong Science and Technology Press. 2004;14(5):2129-2134.

2. Fu Y. Prevention and treatment of the complications after laparoscopic cholecystectomy. The Journal of Practical Medicine. 2006;22(2):130.

3. Sallhamer EA, Sokal SM, Chang Y. Environmental impact of accelerated clinical care in a high-volume center. Surgery. 2007;142(3):343-349.

4. Wang $\mathrm{H}$, Wang $\mathrm{F}$, Zhang $\mathrm{G}$. Clinical analysis of the complications of the 82 cases of laparoscopic surgery in treating urological disease. China Journal of Endoscopy. 2003;12(12):26-27.

5. Yuen TS, Irwin MG. The fifth vital sign. Hong Kong Medical Journal. 2005;11(3):145-146.

6. Xiao B, Wu F. Perioperative nursing of the 28 patients after urological laparoscopic surgery. Nursing Practice and Research. 2009;6(23):59-60. 